\title{
Synthesis, Characterisation, Antimicrobial Evaluation of Chalcones and its Cyclised Product: Phenyl Pyrazolines and Benzodiazepines
}

\author{
ANJANI SOLANKEE* and RIKI TAILOR \\ Department of Chemistry, B. K. M. Science College, Valsad-396001 \\ (Affiliated to the Veer Narmad South Gujarat University, Surat) India \\ dranjani_solankee@yahoo.com
}

Received 2 August 2015 / Accepted 12 August 2015

\begin{abstract}
A conventional root was designed and synthesised for the pyrazolines and benzodiazepines from chalcones. The synthesis of chalcones (7j-n) was accomplished according to the Claisen-Schmidt condensation of substituted acetophenones (C) with various aldehydes. Treatment of this chalcones with phenyl hydrazine hydrochloride / alkali and $o$-phenylenediamine / glacial acetic acid afforded the corresponding 2- phenyl pyrazoline (8j-n) and 1,5- benzodiazepine (9j-n) derivatives respectively. The structures of all the newly synthesised compounds were established based on IR, ${ }^{1} \mathrm{H}$ NMR, mass spectral data as well as elemental analysis. In vitro antimicrobial proficiency of the title compounds were assessed against selected pathogens. Compounds $\mathbf{7 j}, \mathbf{7 m}, \mathbf{7 n}, \mathbf{8 k}, \mathbf{8 m}$ and $\mathbf{9 m}$ exhibited excellent antimicrobial activity and said to be the most proficient members of the series compared to standard drugs.
\end{abstract}

Keywords: Chalcones, Phenyl pyrazolines, Benzodiazepines, Claisen-Schmidt reaction, Antimicrobial activity, Broth dilution method

\section{Introduction}

The most common compounds of chalconoid group are the chalcones, which provide new class of medicines due to the physiologically and pharmacologically active moiety. Chalcones are 1,3-diarylprop-2-en-1-one, form a broad class of compounds containing two aromatic rings which are connected by a three carbon chain ${ }^{1}$. Chalcones were found to have broad spectrum of biological properties such as antiviral ${ }^{2}$, antimalarial ${ }^{3}$, antimicrobial ${ }^{4}$, antitrypanosomacruzi ${ }^{5}$, hepatoprotective ${ }^{6}$ etc.... Hence the synthesis of chalcones has generated huge interest for researcher and chemist to organic as well as medicinal.

Pyrazolines are prominent and significant nitrogen containing five membered heterocyclic compounds and various methods have been worked out for their synthesis. Depending on the reactivity of molecules and need of the chemist, they have synthesised the pyrazolines under various solvent media and acidic or basic conditions ${ }^{7-8}$. It is interesting to 
note that pyrazolines are reported as wellknown several prominent effects such as antimycobacterial $^{9}$, anti-inflammatory ${ }^{10}$, antidepressant ${ }^{11}$, antifeedant ${ }^{12}$, antimicrobial ${ }^{13}$, anticancer $^{14}$ etc... . Benzodiazepines are an important class of nitrogen containing seven membered heterocyclic compounds. Benzodiazepines have importance in medicinal chemistry due to their hopeful and potential pharmaceutical properties ${ }^{15}$. Benzodiazepines have recently received considerable attention because of their promising broad spectrum of biological properties including anticancer ${ }^{16}$, anti-inflammatory ${ }^{17}$, anticonvulsant ${ }^{18}$, antimicrobial ${ }^{19}$ etc... . Keeping in view the importance of these biological activities, it was thought of interest to synthesise some new phenyl pyrazoline and 1,5- benzodiazepine derivatives from chalcones.

\section{Experimental}

All the chemicals and solvents which used for reaction were of analytical reagent (AR) grade. All the melting points were resolute in open capillary method and are uncorrected. Infrared spectra were recorded on Shimadzu FTIR 8401 spectrophotometer using potassium bromide pellets. ${ }^{1} \mathrm{H}$ NMR spectra were recorded on a Bruker Avance DPX $400 \mathrm{MHz}$ spectrometer with $\mathrm{CDCl}_{3}$ as a solvent and TMS as an internal standard. Mass spectra of representative compounds were scanned on a Shimadzu QP 2010 spectrometer. All the compounds were analysed for carbon, hydrogen and nitrogen by the Perkin-Elmer $240 \mathrm{C} \mathrm{H}$ $\mathrm{N}$ elemental analyser. Purity of the compounds were checked by thin layer chromatography using TLC aluminum sheets Silica Gel 60 F-254 (Merck) plates of $0.25 \mathrm{~mm}$ thickness.

\section{General procedure for the preparation of compounds $(A),(B)$ and $(C)$}

Compounds (A), (B) and (C) were prepared by the reported method ${ }^{20}$.

Preparation of 2 - (3' - trifluromethylphenylamino) - 4 - (tetrahydro - 1', 4'- oxazine) - 6 [4' - \{3" - (3, 4'"'- dimethoxyphenyl) - 2" - propenon - $1^{\prime \prime}$ - yl\} phenylamino] - $s$ - triazine (7j)

3,4-Dimethoxy benzaldehyde ( $0.01 \mathrm{~mol}, 1.6 \mathrm{~g}$ in $15 \mathrm{~mL}$ DMF) added into a solution of substituted acetophenone (C) $(0.01 \mathrm{~mol}, 4.5 \mathrm{~g}$ in $20 \mathrm{~mL}$ DMF) in a round-bottomed flask. The solution of $40 \% \mathrm{KOH}(5 \mathrm{~mL})$ was added in it as catalyst to make alkaline. Then the reaction mixture was stirred for 24 hours on a magnetic stirrer at room temprature. The progress of reaction was monitored by TLC. After completion of the reaction, the reaction mixture was poured into crushed ice, neutralised with dilute hydrochloric acid and the mixture was agitated for 4 hours. The product was isolated by filtration and recrystallised from suitable solvent (ethanol) to get pure product. In the same way, the remaining compounds (7k-n) were prepared by this given method.

Preparation of 2 - (3' - trifluromethylphenylamino) - 4 - (tetrahydro - 1', 4' oxazine) - 6 - [4' - \{1"- phenyl 5"- (3, 4"' - dimethoxyphenyl) $2^{\prime \prime}$ - pyrazolin $\left.3{ }^{\prime \prime}-y l\right\}$ phenylamino] - $s$ - triazine (8j)

Chalcone (7j) $(0.01 \mathrm{~mol}, 6.0 \mathrm{~g}$ in $30 \mathrm{~mL}$ ethanol) and phenyl hydrazine hydrochloride $(0.01 \mathrm{~mol}, 1.4 \mathrm{~g}$ in $10 \mathrm{~mL}$ ethanol) was dissolved in alcohol and transfer in round-bottomed flask. The reaction was proceed by the addition of $5 \mathrm{~mL} \mathrm{KOH} \mathrm{(40 \% )} \mathrm{as} \mathrm{basic} \mathrm{solvent}$ medium, then the reaction mixture was refluxed for 6-8 hours. The progress of the reaction was investigated by using TLC. After completion of the reaction, the reaction mixture was poured into crushed ice, neutralise with dilute $\mathrm{HCl}$ and allowed to settle. The solid that separated was collected by filtration, washed with hot water and recrystallised from ethanol to get product $(\mathbf{8 j})$ in good yield with high purity. In the same way, the remaining compounds (8k-n) were prepared by this given process. 
Preparation of 2 - (3' - trifluromethyphenylamino) - 4 - (tetrahydro - 1', 4'- oxazine) - 6 - [4' - \{4"- (3, 4'"'- dimethoxyphenyl) - 3" H - benzo - 1", 5"- diazepin - 2"- yl $\}$ phenylamino] - $s$ - triazine (9j)

Chalcone (7j) $(0.01 \mathrm{~mol}, 5.7 \mathrm{~g})$ and $o$-phenylenediamine $(0.01 \mathrm{~mol}, 1.0 \mathrm{~g})$ dissolved in ethanol $(30 \mathrm{~mL})$ were mixed in round-bottomed flask. To make this mixture acidic $5 \mathrm{~mL}$ glacial aceticacid was added as catalyst, then the reaction mixture was heated under reflux temperature for 5-6 hours. The progress of the reaction was monitored by using TLC. After completion of the reaction, the mixture was cooled at room temperature poured into crushed ice and neutralised with $\mathrm{Na}_{2} \mathrm{CO}_{3}$. Finally, the product was filtered, washed, dried and purified by recrystallisation from ethanol to get product $(\mathbf{9 j})$ in good yield. In the same way other remaining compounds (9k-n) were prepared by this given method.

All the synthesised compounds $(\mathbf{7} \mathbf{j}-\mathbf{n}),(\mathbf{8 j}-\mathbf{n})$ and $(\mathbf{9 j}-\mathbf{n})$ were characterised by $I R,{ }^{1} \mathrm{H}$ NMR and mass spectroscopy as well as elemental analysis. The analytic and spectroscopic data of all the synthesised compounds are given in the spectral analysis data to this paper.

\section{Spectral analysis data}

Spectral and analytical data for the design compounds (7j-n), (8j-n) and (9j-n)

Compound (7j): Yield: 75\%; m. p. $119{ }^{0} \mathrm{C}$; Anal. Calcd. for $\mathrm{C}_{31} \mathrm{H}_{29} \mathrm{~N}_{6} \mathrm{~F}_{3} \mathrm{O}_{4}: \mathrm{C}, 61.38 ; \mathrm{H}$, 4.81; N, 13.86\%. Found: C, 61.42; H, 4.79; N, 13.89\%; IR $\left(\mathrm{KBr}, \mathrm{V}_{\max }, \mathrm{cm}^{-1}\right): 3330(\mathrm{~N}-\mathrm{H}$ str.), 1649 (-C=O str. of $\alpha, \beta$-unsaturated carbonyl group), $1415(-\mathrm{CH}=\mathrm{CH}$ str. of unsaturated carbonyl group), 1561, 1070 (C-F str.), 801 (C-N str. of s-triazine), $695 \& 833$ (C-H bend. of 1,3 and 1,4 disubstituted benzene ring); ${ }^{1} \mathrm{H}$ NMR $\left(400 \mathrm{MHz}, \mathrm{CDCl}_{3}, \delta \mathrm{ppm}\right): 8.01(\mathrm{~s}, 1 \mathrm{H},-$ $\mathrm{NH}), 3.69\left(4 \mathrm{H},-\mathrm{CH}_{2}\right.$, oxazine ring), $3.80\left(\mathrm{t}, 4 \mathrm{H},-\mathrm{CH}_{2}\right.$, oxazine ring), $3.83(\mathrm{~s}, 3 \mathrm{H}, 3-\mathrm{OCH})$, $4.06\left(\mathrm{~s}, 3 \mathrm{H}, 4-\mathrm{OCH}_{3}\right), 6.24(\mathrm{~d}, J=9.87 \mathrm{~Hz}, 1 \mathrm{H},-\mathrm{CO}-\mathrm{CH}=), 8.15(\mathrm{~d}, J=9.56 \mathrm{~Hz}, 1 \mathrm{H}, \mathrm{Ar}-$ $\mathrm{CH}=)$, 7.1-7.9 (m, 11H, Ar-H); M.S. $(\mathrm{m} / \mathrm{z}): 606\left(\mathrm{M}^{+}\right)$.

Compound (7k): Yield: $72 \%$; m. p. $117{ }^{0} \mathrm{C}$; Anal. Calcd. for $\mathrm{C}_{29} \mathrm{H}_{24} \mathrm{~N}_{7} \mathrm{~F}_{4} \mathrm{O}_{3}: \mathrm{C}, 58.88$; $\mathrm{H}, 4.09 ; \mathrm{N}, 16.58 \%$. Found: C, 58.93; H, 4.13; N, 16.61\%; IR (KBr, $\left.\mathrm{v}_{\max ,} \mathrm{cm}^{-1}\right): 3316(\mathrm{~N}-\mathrm{H}$ str.), 1697 (-C=O str. of $\alpha, \beta$-unsaturated carbonyl group), $1543\left(\mathrm{C}-\mathrm{NO}_{2}\right.$ str.), 1010 (C-F str.), 826 (C-H bend. of 1,4 disubstituted benzene ring), 807 (C-N str. of s-triazine); ${ }^{1} \mathrm{H}$ NMR (400 MHz, $\left.\mathrm{CDCl}_{3}, \delta \mathrm{ppm}\right): 7.26(\mathrm{~s}, 1 \mathrm{H},-\mathrm{NH}), 3.71$ (t, $4 \mathrm{H},-\mathrm{CH}_{2}$, oxazine ring), 3.85 (t, 4H, $\mathrm{CH}_{2}$, oxazine ring), $6.89(\mathrm{~d}, J=8.72 \mathrm{~Hz}, 1 \mathrm{H},-\mathrm{CO}-\mathrm{CH}=), 8.23(\mathrm{~d}, J=9.23 \mathrm{~Hz}, 1 \mathrm{H}, \mathrm{Ar}-\mathrm{CH}=)$, 7.1-8.0 (m, 12H, Ar-H); M.S. $(\mathrm{m} / \mathrm{z}): 591\left(\mathrm{M}^{+}\right)$.

Compound (7l): Yield: $68 \%$; m. p. $155{ }^{0} \mathrm{C}$; Anal. Calcd. for $\mathrm{C}_{29} \mathrm{H}_{24} \mathrm{~N}_{7} \mathrm{~F}_{4} \mathrm{O}_{3}:$ C, 58.88; $\mathrm{H}, 4.09$; N, 16.58\%. Found: C, 58.85; H, 4.06; N, 16.57\%; IR $\left(\mathrm{KBr}, \mathrm{v}_{\max }, \mathrm{cm}^{-1}\right): 3305(\mathrm{~N}-\mathrm{H}$ str.), 1678 (-C=O str. of $\alpha, \beta$-unsaturated carbonyl group), 1531 (C-NO ${ }_{2}$ str.), 1008 (C-F str.), 798 (C-N str. of s-triazine), 798 (C-H bend. of 1,3 disubstituted benzene ring); ${ }^{1} \mathrm{H}$ NMR (400 $\left.\mathrm{MHz}, \mathrm{CDCl}_{3}, \delta \mathrm{ppm}\right): 6.98(\mathrm{~s}, 1 \mathrm{H},-\mathrm{NH}), 3.42$ (t, 4H, $-\mathrm{CH}_{2}$, oxazine ring), $3.62\left(\mathrm{t}, 4 \mathrm{H},-\mathrm{CH}_{2}\right.$, oxazine ring), $6.12(\mathrm{~d}, J=9.49 \mathrm{~Hz}, \mathrm{lH},-\mathrm{CO}-\mathrm{CH}=), 8.20(\mathrm{~d}, J=8.93 \mathrm{~Hz}, 1 \mathrm{H}, \mathrm{Ar}-\mathrm{CH}=), 7.1-$ $8.2(\mathrm{~m}, 12 \mathrm{H}, \mathrm{Ar}-\mathrm{H})$; M.S. $(\mathrm{m} / \mathrm{z}): 592\left(\mathrm{M}^{+}\right)$.

Compound (7m): Yield: $67 \%$; m. p. $109{ }^{0} \mathrm{C}$; Anal. Calcd. for $\mathrm{C}_{29} \mathrm{H}_{24} \mathrm{~N}_{6} \mathrm{~F}_{3} \mathrm{O}_{2} \mathrm{Cl}: \mathrm{C}$, 59.95; H, 4.16; N, 14.47\%. Found: C, 59.98; H, 4.13; N, 14.48\%; IR (KBr, $\left.\mathrm{v}_{\max ,} \mathrm{cm}^{-1}\right): 3326$ (N-H str.), 1637 (-C=O str. of $\alpha, \beta$-unsaturated carbonyl group), 1099 (C-F str.), 803 (C-N str. of s-triazine), 702 (C-H bend. of 1,3 disubstituted benzene ring), $656(\mathrm{C}-\mathrm{Cl}$ str. $) ;{ }^{1} \mathrm{H}$ NMR (400 MHz, $\left.\mathrm{CDCl}_{3}, \delta \mathrm{ppm}\right): 8.4(\mathrm{~s}, 1 \mathrm{H},-\mathrm{NH}), 3.51$ (t, $4 \mathrm{H},-\mathrm{CH}_{2}$, oxazine ring), 3.81 (t, $4 \mathrm{H},-\mathrm{CH}_{2}$, oxazine ring), $6.85(\mathrm{~d}, J=8.71 \mathrm{~Hz}, 1 \mathrm{H},-\mathrm{CO}-\mathrm{CH}=), 8.14(\mathrm{~d}, J=9.19 \mathrm{~Hz}, 1 \mathrm{H}, \mathrm{Ar}-$ $\mathrm{CH}=)$, 7.2-7.8 (m, 12H, Ar-H); M.S. $(\mathrm{m} / \mathrm{z}): 579\left(\mathrm{M}^{+}\right)$. 
Compound (7n): Yield 72\%; m. p. $127{ }^{0} \mathrm{C}$; Anal. Calcd. for $\mathrm{C}_{27} \mathrm{H}_{23} \mathrm{~N}_{6} \mathrm{~F}_{3} \mathrm{O}_{2} \mathrm{~S}$ : C, 58.69; H, 4.19; N, 15.21\%. Found: C, 58.67; H, 4.21; N, 15.24\%; IR (KBr, $\left.\mathrm{v}_{\max } \mathrm{cm}^{-1}\right): 3323(\mathrm{~N}-\mathrm{H}$ str.), 1634 (-C=O str. of $\alpha, \beta$-unsaturated carbonyl group), 1020 (C-F str.), 803 (C-N str. of striazine), 666 (C-S-C str. of sulphur linkage); ${ }^{1} \mathrm{H}$ NMR (400 MHz, $\mathrm{CDCl}_{3}, \delta \mathrm{ppm}$ ): 7.20 (s, $1 \mathrm{H},-\mathrm{NH}), 3.39$ (t, $4 \mathrm{H},-\mathrm{CH}_{2}$, oxazine ring), 3.52 (t, $4 \mathrm{H},-\mathrm{CH}_{2}$, oxazine ring), 6.93 (d, $J=$ $8.58 \mathrm{~Hz}, 1 \mathrm{H},-\mathrm{CO}-\mathrm{CH}=), 8.78(\mathrm{~d}, J=8.73 \mathrm{~Hz}, 1 \mathrm{H}, \mathrm{Ar}-\mathrm{CH}=), 7.0-8.0(\mathrm{~m}, 11 \mathrm{H}, \mathrm{Ar}-\mathrm{H})$; M.S. $(\mathrm{m} / \mathrm{z}): 552\left(\mathrm{M}^{+}\right)$.

Compound (8j): Yield: $72 \%$; m. p. $115{ }^{0} \mathrm{C}$; Anal. Calcd. for $\mathrm{C}_{37} \mathrm{H}_{35} \mathrm{~N}_{8} \mathrm{~F}_{3} \mathrm{O}_{3}$ : C, 63.79;

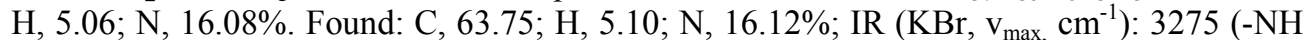
str.), 2931 (C-H str. of pyrazoline), 1572 (C=N, str. of pyrazoline), 1099 (C-F str.), 809 (C-N str. of s-triazine), $690 \& 840$ (C-H bend. of 1,3 and 1,4 disubstituted benzene ring); ${ }^{1} \mathrm{H}$ NMR (400 MHz, $\left.\mathrm{CDCl}_{3}, \delta \mathrm{ppm}\right): 2.2\left(\mathrm{dd}, J=11.5 \& 13.0 \mathrm{~Hz}, 1 \mathrm{H},-\mathrm{CH}^{\mathrm{x}}-\mathrm{CH}\right), 3.2(\mathrm{dd}, J=$ $\left.11.3 \& 13.0 \mathrm{~Hz}, 1 \mathrm{H},-\underline{\mathrm{H}}^{\mathrm{y}}-\mathrm{CH}\right), 5.1\left(\mathrm{dd}, J=4.6 \& 11.8 \mathrm{~Hz}, 1 \mathrm{H},-\mathrm{C} \underline{\mathrm{H}}-\mathrm{CH}_{2}-\mathrm{Ar}\right), 3.72(\mathrm{~s}, 3 \mathrm{H}$, 3- $\left.\mathrm{OCH}_{3}\right), 3.82\left(\mathrm{~s}, 3 \mathrm{H}, 4-\mathrm{OCH}_{3}\right), 3.68\left(\mathrm{t}, 4 \mathrm{H},-\mathrm{CH}_{2}\right.$, oxazine ring), 3.89 (t, $4 \mathrm{H},-\mathrm{CH}_{2}$, oxazine ring), 6.9-8.3 (m, 17H, 16 Ar- $\underline{\mathrm{H}}$ and 1-N$)$; M.S. $(\mathrm{m} / \mathrm{z}): 696\left(\mathrm{M}^{+}\right)$.

Compound (8k): Yield: 59\%; m. p. $159{ }^{\circ} \mathrm{C}$; Anal. Calcd. for $\mathrm{C}_{35} \mathrm{H}_{30} \mathrm{~N}_{9} \mathrm{~F}_{3} \mathrm{O}_{3}:$ C, 61.67; H, 4.43; N, 18.49\%. Found: C, 61.63; H, 4.42; N, 18.48\%; IR (KBr, $\left.\mathrm{v}_{\max }, \mathrm{cm}^{-1}\right): 3307$ (-NH str.), 3013 (C-H str. of pyrazoline), 1569 (C=N str. of pyrazoline), $1479\left(\mathrm{C}-\mathrm{NO}_{2}\right.$ str.), 1070 (C-F str.), 839 (C-H bend. of 1,4 disubstituted benzene ring), 796 (C-N str. of s-triazine); ${ }^{1} \mathrm{H}$ NMR $\left(400 \mathrm{MHz}, \mathrm{CDCl}_{3}, \delta \mathrm{ppm}\right): 1.9\left(\mathrm{dd}, J=11.2 \& 13.3 \mathrm{~Hz}, 1 \mathrm{H},-\mathrm{C}^{\mathrm{x}}-\mathrm{CH}\right), 2.3(\mathrm{dd}, J=$ $\left.11.5 \& 13.2 \mathrm{~Hz}, 1 \mathrm{H},-\underline{\mathrm{H}}^{\mathrm{y}}-\mathrm{CH}\right), 5.3\left(\mathrm{dd}, J=5.0 \& 12.0 \mathrm{~Hz}, 1 \mathrm{H},-\mathrm{C} \underline{\mathrm{H}}-\mathrm{CH}_{2}-\mathrm{Ar}\right.$ ), 3.49 (t, $4 \mathrm{H},-$ $\mathrm{CH}_{2}$, oxazine ring), 3.65 (t, $4 \mathrm{H},-\mathrm{CH}_{2}$, oxazine ring), 7.0-8.4 (m, $18 \mathrm{H}, 17 \mathrm{Ar}-\underline{\mathrm{H}}$ and 1-N $\left.\underline{\mathrm{H}}\right)$; M.S. $(\mathrm{m} / \mathrm{z}): 680\left(\mathbf{M}^{+}\right)$.

Compound (8I): Yield: 69\%; m. p. $127{ }^{0} \mathrm{C}$; Anal. Calcd. for $\mathrm{C}_{35} \mathrm{H}_{30} \mathrm{~N}_{9} \mathrm{~F}_{3} \mathrm{O}_{3}$ : C, 61.67; $\mathrm{H}, 4.43 ; \mathrm{N}, 18.49 \%$. Found: C, 61.66; H, 4.39; N, 18.51\%; IR (KBr, $\left.\mathrm{v}_{\max } \mathrm{cm}^{-1}\right): 3310(-\mathrm{NH}$ str.), 3009 (C-H str. of pyrazoline), $1579(\mathrm{C}=\mathrm{N}$ str. of pyrazoline $), 1496\left(\mathrm{C}-\mathrm{NO}_{2}\right.$ str. $), 1054$ (C-F str.), 802 (C-N str. of s-triazine), 712 (C-H bend. of 1,3 disubstituted benzene ring); ${ }^{1} \mathrm{H}$ NMR (400 MHz, $\left.\mathrm{CDCl}_{3}, \delta \mathrm{ppm}\right): 1.8\left(\mathrm{dd}, J=11.7 \& 12.8 \mathrm{~Hz}, 1 \mathrm{H},-\mathrm{CH}^{\mathrm{x}}-\mathrm{CH}\right), 2.2(\mathrm{dd}, J=$ $\left.11.3 \& 12.7 \mathrm{~Hz}, 1 \mathrm{H},-\mathrm{CH}^{\mathrm{y}}-\mathrm{CH}\right), 5.0\left(\mathrm{dd}, J=4.9 \& 12.5 \mathrm{~Hz}, 1 \mathrm{H},-\mathrm{C} \underline{\mathrm{H}}-\mathrm{CH}_{2}-\mathrm{Ar}\right), 3.63(\mathrm{t}, 4 \mathrm{H},-$ $\mathrm{CH}_{2}$, oxazine ring), 3.90 (t, $4 \mathrm{H},-\mathrm{CH}_{2}$, oxazine ring), 6.9-8.2 (m, $18 \mathrm{H}, 17 \mathrm{Ar}-\underline{\mathrm{H}}$ and $\left.1-\mathrm{N} \underline{\mathrm{H}}\right)$; M.S. $(\mathrm{m} / \mathrm{z}): 681\left(\mathrm{M}^{+}\right)$.

Compound (8m): Yield: 64\%; m. p. $120{ }^{0} \mathrm{C}$; Anal. Calcd. for $\mathrm{C}_{35} \mathrm{H}_{30} \mathrm{~N}_{8} \mathrm{~F}_{3} \mathrm{OCl}$ : C, 62.64; H, 4.50; N, 16.70\%. Found: C, 62.67; H, 4.53; N, 16.72\%; IR (KBr, $\left.v_{\max } \mathrm{cm}^{-1}\right): 3159$ (-NH str.), 2921 (C-H str. of pyrazoline), 1629 (C=N str. of pyrazoline), 1059 (C-F str.), 806 (C-N str. of s-triazine), 689 (C-H bend. of 1,3 disubstituted benzene ring), 639 (C-Cl str.); ${ }^{1} \mathrm{H}$ NMR (400 MHz, $\left.\mathrm{CDCl}_{3}, \delta \mathrm{ppm}\right): 2.2\left(\mathrm{dd}, J=10.9 \& 11.5 \mathrm{~Hz}, 1 \mathrm{H},-\mathrm{C}^{\mathrm{x}}-\mathrm{CH}\right), 2.6(\mathrm{dd}, J$ $\left.=10.7 \& 11.9 \mathrm{~Hz}, 1 \mathrm{H},-\underline{\mathrm{C}}^{\mathrm{y}}-\mathrm{CH}\right), 4.9\left(\mathrm{dd}, J=4.4 \& 12.1 \mathrm{~Hz}, 1 \mathrm{H},-\mathrm{CH}_{-}-\mathrm{CH}_{2}-\mathrm{Ar}\right), 3.74(\mathrm{t}, 4 \mathrm{H}$, $-\mathrm{CH}_{2}$, oxazine ring), 3.93 (t, $4 \mathrm{H},-\mathrm{CH}_{2}$, oxazine ring), 7.2-8.5 (m, 18H, $17 \mathrm{Ar}-\underline{\mathrm{H}}$ and 1-N $\underline{\mathrm{H}}$ ); M.S. $(\mathrm{m} / \mathrm{z}): 671\left(\mathrm{M}^{+}\right)$.

Compound (8n): Yield: 71\%; m. p. $123{ }^{\circ} \mathrm{C}$; Anal. Calcd. for $\mathrm{C}_{33} \mathrm{H}_{29} \mathrm{~N}_{8} \mathrm{~F}_{3} \mathrm{OS}$ : C, 61.67; $\mathrm{H}, 4.54 ; \mathrm{N}, 17.44 \%$. Found: C, 61.65; H, 4.57; N, 17.40\%; IR (KBr, $\left.\mathrm{v}_{\max } \mathrm{cm}^{-1}\right): 3243(-\mathrm{NH}$ str.), 2910 (C-H str. of pyrazoline), 1523 (C=N str. of pyrazoline), 1069 (C-F str.), 799 (C-N str. of s-triazine), 651 (C-S-C str. of sulphur linkage); ${ }^{1} \mathrm{H}$ NMR $\left(400 \mathrm{MHz}, \mathrm{CDCl}_{3}, \delta \mathrm{ppm}\right)$ : $2.3\left(\mathrm{dd}, J=11.6 \& 14.5 \mathrm{~Hz}, 1 \mathrm{H},-\mathrm{CH}^{\mathrm{x}}-\mathrm{CH}\right), 2.5\left(\mathrm{dd}, J=11.6 \& 14.3 \mathrm{~Hz}, 1 \mathrm{H},-\underline{\mathrm{C}}^{\mathrm{y}}-\mathrm{CH}\right), 5.2$ (dd, $J=5.9 \& 14.2 \mathrm{~Hz}, 1 \mathrm{H},-\mathrm{C} \underline{\mathrm{H}}-\mathrm{CH}_{2}-\mathrm{Ar}$ ), 3.39 (t, $4 \mathrm{H},-\mathrm{CH}_{2}$, oxazine ring), 3.61 (t, $4 \mathrm{H}$, $-\mathrm{CH}_{2}$, oxazine ring), 6.8-8.3 (m, 17H, $16 \mathrm{Ar}-\underline{\mathrm{H}}$ and 1-N$)$; M.S. $(\mathrm{m} / \mathrm{z}): 641\left(\mathrm{M}^{+}\right)$. 
Compound (9j): Yield: 68\%; m. p. $107{ }^{0} \mathrm{C}$; Anal. Calcd. for $\mathrm{C}_{37} \mathrm{H}_{33} \mathrm{~N}_{8} \mathrm{~F}_{3} \mathrm{O}_{3}$ : C, 63.97; H, 4.78; N, 16.13\%. Found: C, 63.99; H, 4.74; N, 16.10\%; IR (KBr, $\left.\mathrm{v}_{\max }, \mathrm{cm}^{-1}\right): 3359(-\mathrm{NH}$ str.), 1589 (C=N str. of benzodiazepine), 1110 (C-F str.), 809 (C-N str. of s-triazine), 716 \& 823 (C-H bend. of 1,3 and 1,4 disubstituted benzene ring); ${ }^{1} \mathrm{H}$ NMR (400 $\mathrm{MHz}, \mathrm{CDCl}_{3}, \delta$ ppm): 2.68 (dd, $J=6.2 \& 13.8 \mathrm{~Hz}, 1 \mathrm{H},-\mathrm{CH}^{\mathrm{x}}-\mathrm{C}$, benzodiazepine), 3.51 (dd, $J=6.2 \& 14.2$ $\mathrm{Hz}, 1 \mathrm{H},-\mathrm{CH}^{\mathrm{y}}-\mathrm{C}$, benzodiazepine), $3.92\left(\mathrm{~s}, 3 \mathrm{H}, 3-\mathrm{OCH}_{3}\right), 4.12\left(\mathrm{~s}, 3 \mathrm{H}, 4-\mathrm{OCH}_{3}\right), 3.61(\mathrm{t}, 4 \mathrm{H}$, $-\mathrm{CH}_{2}$, oxazine ring), 3.79 (t, $4 \mathrm{H},-\mathrm{CH}_{2}$, oxazine ring), 6.9-8.0 (m, 15H, Ar- $\left.\mathrm{H}\right), 8.4(\mathrm{Ar}-\mathrm{N} \underline{\mathrm{H}})$; M.S. $(\mathrm{m} / \mathrm{z}): 693\left(\mathrm{M}^{+}\right)$.

Compound (9k): Yield: 78\%; m. p. $107{ }^{0} \mathrm{C}$; Anal. Calcd. for $\mathrm{C}_{35} \mathrm{H}_{28} \mathrm{~N}_{9} \mathrm{~F}_{3} \mathrm{O}_{3}$ : C, 61.85; H, 4.15; N, 18.55\%. Found: C, 61.89; H, 4.13; N, 18.58\%; IR (KBr, $\left.\mathrm{v}_{\max } \mathrm{cm}^{-1}\right): 3299(-\mathrm{NH}$ str.), 1639 ( $\mathrm{C}=\mathrm{N}$ str. of benzodiazepine), $1476\left(\mathrm{C}-\mathrm{NO}_{2}\right.$ str. $), 1089$ (C-F str.), 834 (C-H bend. of 1,4 disubstituted benzene ring), 800 (C-N str. of s-triazine); ${ }^{1} \mathrm{H}$ NMR (400 MHz, $\mathrm{CDCl}_{3}$, $\delta \mathrm{ppm}): 2.93\left(\mathrm{dd}, J=6.4 \& 13.3 \mathrm{~Hz}, 1 \mathrm{H},-\mathrm{CH}^{\mathrm{x}}-\mathrm{C}\right.$, benzodiazepine), 3.63 (dd, $J=6.7 \& 13.3$ $\mathrm{Hz}, \quad 1 \mathrm{H},-\mathrm{C}^{\mathrm{y}}-\mathrm{C}$, benzodiazepine), $3.55\left(\mathrm{t}, 4 \mathrm{H},-\mathrm{CH}_{2}\right.$, oxazine ring), $3.84\left(\mathrm{t}, 4 \mathrm{H},-\mathrm{CH}_{2}\right.$,

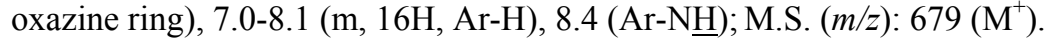

Compound (9l): Yield: $67 \%$; m. p. $140{ }^{0} \mathrm{C}$; Anal. Calcd. for $\mathrm{C}_{35} \mathrm{H}_{28} \mathrm{~N}_{9} \mathrm{~F}_{3} \mathrm{O}_{3}$ : C, 61.85; H, 4.15; N, 18.55\%. Found: C, 61.88; H, 4.18; N, 18.53\%; IR (KBr, $\left.\mathrm{v}_{\max } \mathrm{cm}^{-1}\right): 3311(-\mathrm{NH}$ str.), 1461 (C-NO ${ }_{2}$ str.), 1644 (C=N str. of benzodiazepine), 1082 (C-F str.), 802 (C-N str. of s-triazine), 706 (C-H bend. of 1,3 disubstituted benzene ring); ${ }^{1} \mathrm{H}$ NMR (400 $\mathrm{MHz}, \mathrm{CDCl}_{3}, \delta$ ppm): 2.75 (dd, $J=7.0 \& 12.5 \mathrm{~Hz}, 1 \mathrm{H},-\mathrm{CH}^{\mathrm{x}}-\mathrm{C}$, benzodiazepine), $3.92(\mathrm{dd}, J=7.1 \& 12.4$ $\mathrm{Hz}, 1 \mathrm{H},-\mathrm{CH}^{\mathrm{y}}-\mathrm{C}$, benzodiazepine), $3.47\left(\mathrm{t}, 4 \mathrm{H},-\mathrm{CH}_{2}\right.$, oxazine ring), $3.71\left(\mathrm{t}, 4 \mathrm{H},-\mathrm{CH}_{2}\right.$, oxazine ring), 7.1-8.0 (m, 16H, Ar-H), 8.2 (Ar-N $\underline{H})$; M.S. (m/z): $680\left(\mathrm{M}^{+}\right)$.

Compound (9m): Yield: $76 \%$; m. p. $110{ }^{0} \mathrm{C}$; Anal. Calcd. for $\mathrm{C}_{35} \mathrm{H}_{28} \mathrm{~N}_{8} \mathrm{~F}_{3} \mathrm{OCl}$ : C, 62.83; H, 4.21; N, 16.75\%. Found: C, 62.78; H, 4.17; N, 16.71\%; IR (KBr, $\left.\mathrm{v}_{\max } \mathrm{cm}^{-1}\right): 3319$ (-NH str.), 1636 (C=N str. of benzodiazepine), 1048 (C- F str.), 803 (C-N str. of s-triazine), 695 (C-H bend. of 1,3 disubstituted benzene ring), 689 (C-Cl str.); ${ }^{1} \mathrm{H}$ NMR (400 MHz, $\left.\mathrm{CDCl}_{3}, \delta \mathrm{ppm}\right): 2.31$ (dd, $J=6.9 \& 13.4 \mathrm{~Hz}, 1 \mathrm{H},-\mathrm{CH}^{\mathrm{x}}-\mathrm{C}$, benzodiazepine), 3.04 (dd, $J=6.8$ \& $13.4 \mathrm{~Hz}, 1 \mathrm{H},-\mathrm{CH}^{\mathrm{y}}-\mathrm{C}$, benzodiazepine), $3.54\left(\mathrm{t}, 4 \mathrm{H},-\mathrm{CH}_{2}\right.$, oxazine ring), $3.76(\mathrm{t}, 4 \mathrm{H},-$

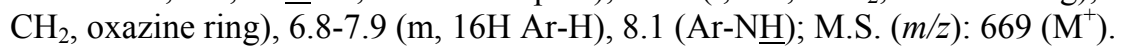

Compound (9n): Yield: 73\%; m. p. $102{ }^{0} \mathrm{C}$; Anal. Calcd. for $\mathrm{C}_{33} \mathrm{H}_{27} \mathrm{~N}_{8} \mathrm{~F}_{3} \mathrm{OS}$ : C, 61.87; H, 4.24; N, 17.49\%. Found: C, 61.83; H, 4.21; N, 17.46\%; IR (KBr, $\left.\mathrm{v}_{\max } \mathrm{cm}^{-1}\right): 3345(-\mathrm{NH}$ str.), 1644 ( $\mathrm{C}=\mathrm{N}$ str. of benzodiazepine), 1099 (C- F str.), 803 (C-N str. of s-triazine), 639 (C-S-C str. of sulphur linkage); ${ }^{1} \mathrm{H}$ NMR $\left(400 \mathrm{MHz}, \mathrm{CDCl}_{3}, \delta \mathrm{ppm}\right): 3.48$ (dd, $J=5.9 \&$ $12.3 \mathrm{~Hz}, 1 \mathrm{H},-\mathrm{C}^{\mathrm{x}}-\mathrm{C}$, benzodiazepine), 3.69 (dd, $J=6.1 \& 12.2 \mathrm{~Hz}, 1 \mathrm{H},-\mathrm{CH}^{\mathrm{y}}-\mathrm{C}$, benzodiazepine ), $3.62\left(\mathrm{t}, 4 \mathrm{H},-\mathrm{CH}_{2}\right.$, oxazine ring), $3.81\left(\mathrm{t}, 4 \mathrm{H},-\mathrm{CH}_{2}\right.$, oxazine ring), 7.1-8.2 (m, 15H, Ar-H), 8.4 (Ar-N $\underline{\mathrm{H}})$; M.S. $(\mathrm{m} / \mathrm{z}): 640\left(\mathrm{M}^{+}\right)$.

\section{Results and Discussion}

\section{Chemistry}

Methodical synthetic route for the target compounds (7j-n), (8j-n) and (9j-n) is outline in Scheme 1. The aim of the present study is to develop an efficient protocol to obtain 1,3- diaryl-2-propen-1-ones (chalcones) and convert them into its cyclised products with good to excellent yield in a short span of time without formation of any side product. The formation of all the synthesised compounds was confirmed by their IR, ${ }^{1} \mathrm{H}$ NMR and mass spectral data. The IR spectrum of compound $7 \mathbf{j}$ shows the characteristic band at $1649 \mathrm{~cm}^{-1}$ due to $>\mathrm{C}=\mathrm{O}$ group of chalcone moiety, while the IR spectrum of compound $\mathbf{8 j}$ and $\mathbf{9 j}$ shows the 
characteristic band at 1572 and $1583 \mathrm{~cm}^{-1}$ due to the $-\mathrm{C}=\mathrm{N}$ group of pyrazoline and benzodiazepine moiety. There are no absorptions in the region of $1600-1700 \mathrm{~cm}^{-1}$ in IR spectra of compound $8 \mathbf{j}$ and $9 \mathbf{j}$ that indicating the absence of $\mathrm{a}>\mathrm{C}=\mathrm{O}$ group in these structures. The ${ }^{1} \mathrm{H}$ NMR spectrum of compound 7j showed doublet of $-\mathrm{CO}-\mathrm{CH}=$ at $\delta 6.24 \mathrm{ppm}(J=9.87 \mathrm{~Hz})$ and doublet of $\mathrm{Ar}-\mathrm{CH}=$ at $\delta 8.15 \mathrm{ppm}(J=9.56 \mathrm{~Hz})$, which confirm the presence of chalcone moiety. The ${ }^{1} \mathrm{H}$ NMR spectrum of compound 8j showed double doublet of $-\mathrm{CH}^{\mathrm{x}}-\mathrm{CH}$ at $\delta 2.2$ $\operatorname{ppm}(J=11.5 \& 13.0 \mathrm{~Hz}),-\underline{\mathrm{C}}^{\mathrm{y}}-\mathrm{CH}$ at $\delta 3.2 \mathrm{ppm}(J=11.3 \& 13.0 \mathrm{~Hz})$ and $-\mathrm{C} \underline{\mathrm{H}}-\mathrm{CH}_{2}-\mathrm{Ar}$ at $\delta$ $5.1 \mathrm{ppm}(J=4.6 \& 11.8 \mathrm{~Hz})$ which confirm the cyclisation of pyrazoline moiety. The ${ }^{1} \mathrm{H}$ NMR spectrum of compound $9 \mathbf{j}$ showed double doublet of $-\mathrm{CH}^{\mathrm{x}}-\mathrm{C}$ at $\delta 2.68 \mathrm{ppm}(J=6.2 \& 13.8$ $\mathrm{Hz}),-\underline{\mathrm{H}}^{\mathrm{y}}-\mathrm{C}$ at $\delta 3.51 \mathrm{ppm}(J=6.2 \& 14.2 \mathrm{~Hz})$ which confirm the cyclisation of benzodiazepine moiety. The aromatic clusters of all the designed compounds also support the synthesis of compounds. Further, the mass spectrum of compound $\mathbf{7 j}, \mathbf{8 j}$ and $\mathbf{9 j}$ shows $\mathrm{M}^{+}$ $(100 \%)$ at $\mathrm{m} / \mathrm{z} 606,696$ and 693 respectively along with other fragment peaks.<smiles>Nc1cccc(C(F)(F)F)c1</smiles><smiles>FC(F)(F)c1cccc(Nc2nc(Cl)c(Cl)c(Cl)n2)c1</smiles><smiles>O=C1CNCCO1</smiles><smiles>FC(F)(F)c1cccc(Nc2nc(Cl)nc(N3CCOCC3)n2)c1</smiles><smiles>CC(=O)c1ccc(Nc2nc(Nc3cccc(C(F)(F)F)c3)nc(N3CCOCC3)n2)cc1</smiles>

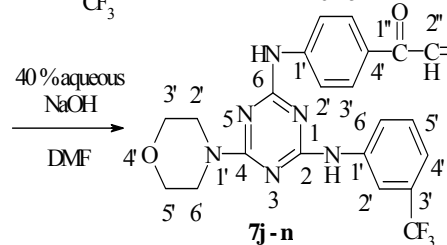
$\stackrel{\mathrm{C}}{\mathrm{H}}-\mathrm{R}$ $\mathrm{CF}_{3}$
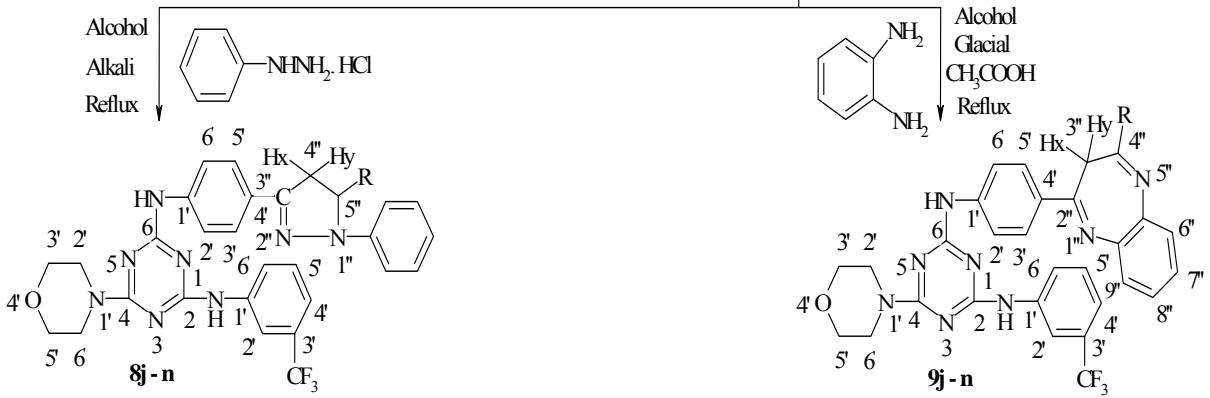

Scheme 1. Methodical synthetic route for the target compounds (7j-n), (8j-n) and (9j-n)

\section{Biological result}

The antibacterial and antifungal activity of newly designed compounds (7j-n, $\mathbf{8 j}-\mathbf{n}$ and $\mathbf{9 j}$-n) was carried out by micro broth dilution method ${ }^{20}$ according to National Committee for Clinical Laboratory Standards (NCCLS, 2002). Upon reviewing antimicrobial data (Table 1) it has been observed that in Gram positive bacterial strains, compounds $7 \mathbf{j}$ and $8 \mathbf{k}$ (MIC $=62.5$ $\mu \mathrm{g} / \mathrm{mL}$ ) exhibited an outstanding inhibitory effect against Staphylococcus aureus compared to Ampicillin ( $\mathrm{MIC}=250 \mu \mathrm{g} / \mathrm{mL}$ ) and modest to Chloramphenicol and Ciprofloxacin (MIC $=50 \mu \mathrm{g} / \mathrm{mL})$ whereas compounds $7 \mathbf{l}, 7 \mathbf{m}$ and $9 \mathbf{k}(\mathrm{MIC}=100 \mu \mathrm{g} / \mathrm{mL})$ showed significant activity against Staphylococcus aureus compared to Ampicillin (MIC $=250 \mu \mathrm{g} / \mathrm{mL}$ ) and moderate to Chloramphenicol and Ciprofloxacin ( $\mathrm{MIC}=50 \mu \mathrm{g} / \mathrm{mL}$ ). Against Streptococcus 
pyogenes, compounds $\mathbf{7 m}$ and $\mathbf{9 m}(\mathrm{MIC}=62.5 \mu \mathrm{g} / \mathrm{mL}$ ) found to possesses excellent activity compared to Ampicillin ( $\mathrm{MIC}=100 \mu \mathrm{g} / \mathrm{mL}$ ) while compounds 7k, 7n, 8n and 9l (MIC = $100 \mu \mathrm{g} / \mathrm{mL}$ ) found equipotent to Ampicillin ( $\mathrm{MIC}=100 \mu \mathrm{g} / \mathrm{mL}$ ) and less potent to Chloramphenicol and Ciprofloxacin ( $\mathrm{MIC}=50 \mu \mathrm{g} / \mathrm{mL})$. In Gram negative bacterial strains, compound $8 \mathbf{m}(\mathrm{MIC}=50 \mu \mathrm{g} / \mathrm{mL})$ and $7 \mathbf{n}(\mathrm{MIC}=62.5 \mu \mathrm{g} / \mathrm{mL})$ exhibited maximum activity compared to Ampicillin (MIC $=100 \mu \mathrm{g} / \mathrm{mL}$ ) while compounds $\mathbf{7 m}, \mathbf{9 m}$ and $\mathbf{9 n}$ (MIC $=100$ $\mu \mathrm{g} / \mathrm{mL}$ ) showed same potency to Ampicillin (MIC $=100 \mu \mathrm{g} / \mathrm{mL}$ ) against Escherichia coli. Compounds 8n, 9j and 9n ( $\mathrm{MIC}=100 \mu \mathrm{g} / \mathrm{mL})$ exerted equivalent activity to Ampicillin $(\mathrm{MIC}=100 \mu \mathrm{g} / \mathrm{mL})$ and mild to Chloramphenicol $(\mathrm{MIC}=50 \mu \mathrm{g} / \mathrm{mL})$ against Pseudomonas aeruginosa.

The antifungal screening data (Table 1) revealed that compounds $8 \mathrm{n}$ and $\mathbf{9 m}$ (MIC $=$ $100 \mu \mathrm{g} / \mathrm{mL})$ and $9 \mathbf{n}(\mathrm{MIC}=250 \mu \mathrm{g} / \mathrm{mL})$ displayed outstanding inhibitory effect compared to Greseofulvin ( $\mathrm{MIC}=500 \mu \mathrm{g} / \mathrm{mL}$ ) and good to Nystatin $(\mathrm{MIC}=100 \mu \mathrm{g} / \mathrm{mL}$ ) against Candida albicans whereas compounds $\mathbf{7 j}, \mathbf{7 k}, \mathbf{7 m}, \mathbf{8 j}, \mathbf{8 m}$ and $\mathbf{9 k}(\mathrm{MIC}=500 \mu \mathrm{g} / \mathrm{mL})$ exerted equipotent to Greseofulvin ( $\mathrm{MIC}=500 \mu \mathrm{g} / \mathrm{mL}$ ) against Candida albicans. None of the compound showed promising antifungal activity against Aspergillus niger and Aspergillus clavatus.

Table 1. Antimicrobial activity of the compounds (7j-n), (8j-n) and (9j-n)

\begin{tabular}{|c|c|c|c|c|c|c|c|}
\hline \multirow[b]{3}{*}{ Compd. } & \multicolumn{4}{|c|}{$\begin{array}{c}\text { Minimal bactericidal } \\
\text { Concentration } \mathrm{MIC}-\mu \mathrm{g} / \mathrm{mL}\end{array}$} & \multicolumn{3}{|c|}{$\begin{array}{c}\text { Minimal fungicidal } \\
\text { concentration MIC }-\mu \mathrm{g} / \mathrm{mL}\end{array}$} \\
\hline & \multicolumn{2}{|c|}{ Gram positive } & \multicolumn{2}{|c|}{ Gram negative } & & & \\
\hline & $\begin{array}{l}\text { S. } a \text { MTCC } \\
96\end{array}$ & $\begin{array}{c}S . p \\
\text { MTCC } \\
442\end{array}$ & $\begin{array}{c}E . c \\
\text { MTCC } \\
443\end{array}$ & $\begin{array}{c}P . a \\
\text { MTCC } \\
441\end{array}$ & $\begin{array}{c}\text { C. } a \\
\text { MTCC } \\
227\end{array}$ & $\begin{array}{c}A . n \\
\text { MTCC } \\
227\end{array}$ & $\begin{array}{c}A . c \\
\text { MTCC } \\
227\end{array}$ \\
\hline $7 \mathbf{j}$ & 62.5 & 200 & 250 & 200 & 500 & 500 & 250 \\
\hline $7 \mathbf{k}$ & 200 & 100 & 200 & 200 & 500 & $>1000$ & 500 \\
\hline 71 & 100 & 200 & 250 & 200 & $>1000$ & $>1000$ & $>1000$ \\
\hline $7 \mathrm{~m}$ & 100 & 62.5 & 100 & 250 & 500 & 1000 & 200 \\
\hline $7 n$ & 125 & 100 & 62.5 & 200 & 1000 & 500 & $>1000$ \\
\hline $8 \mathbf{j}$ & 200 & 200 & 200 & 200 & 500 & 500 & 500 \\
\hline $8 \mathbf{k}$ & 62.5 & 250 & 200 & 250 & $>1000$ & $>1000$ & $>1000$ \\
\hline 81 & 250 & 125 & 125 & 200 & 1000 & 500 & $>1000$ \\
\hline $8 m$ & 250 & 200 & 50 & 250 & 500 & 500 & 500 \\
\hline $8 n$ & 250 & 100 & 500 & 100 & 100 & 250 & 500 \\
\hline 9j & 125 & 200 & 500 & 100 & 1000 & $>1000$ & $>1000$ \\
\hline $9 \mathbf{k}$ & 100 & 200 & 125 & 250 & 500 & $>1000$ & $>1000$ \\
\hline 91 & 200 & 100 & 200 & 200 & 1000 & $>1000$ & $>1000$ \\
\hline $9 m$ & 250 & 62.5 & 100 & 125 & 100 & 500 & 1000 \\
\hline $9 n$ & 250 & 250 & 100 & 100 & 250 & 500 & 1000 \\
\hline Ampi. & 250 & 100 & 100 & 100 & - & - & - \\
\hline Chlo. & 50 & 50 & 50 & 50 & - & - & - \\
\hline Cipr. & 50 & 50 & 25 & 25 & - & - & - \\
\hline Gris. & - & - & - & - & 500 & 100 & 100 \\
\hline Nyst. & - & - & - & - & 100 & 100 & 100 \\
\hline
\end{tabular}




\section{Conclusion}

In conclusion, the present work demonstrates the successfully two elegent protocols have been developed using the potential of chalcones. The method adopted for the synthesis of pharmacologically important molecules in this investigation is simple, efficient and inexpensive. The IR, ${ }^{1} \mathrm{H}$ NMR, mass spectral analysis and elemental analysis of all the newly synthesised compounds confirmed that purity of the entire synthesised compound is good. All the synthesised compounds were screened for antimicrobial activity. Reviewing the antimicrobial data, majority of the synthesised compounds were found to potentially active against both selected Gram positive, Gram negative organisms and selected fungal organisms. Antimicrobial screening results revealed that compounds $\mathbf{7 j}, \mathbf{7 m}, \mathbf{7 n}, \mathbf{8 k}, \mathbf{8 m}$ and 9m were found to be the most proficient members of the series which indicate the introduction of electrophilic substituent such as methoxy, chlorine on the compounds shows a excellent activity. These result suggest that chalcones and their derivatives have an opportunity to behave as generation of newer antimicrobial agents and have exellent scope for further development as commercial antimicrobial agents.

\section{Acknowledgement}

Authors are grateful to B. K. M. Science College, Valsad for providing research facilities, Atul Ltd. (Atul) for the IR analysis, RSIC Punjab University for the ${ }^{1} \mathrm{H}$ NMR, mass spectral analysis as well as elemental analysis and Microcare Laboratory, Surat, for antimicrobial activity screening.

\section{References}

1. Rane R A and Telvekar V N, Bioorg Med Chem Lett., 2010, 20(19), 5681-5685; DOI:10.1016/j.bmcl.2010.08.026

2. Trivedi J C, Bariwal J B, Upadhyay K D, Naliapara Y T, Joshi S K, Pannecouque C C, Clercq E D and Shah A K, Tetrahedron Lett., 2007, 48, 8472-8474; DOI:10.1016/j.tetlet.2007.09.175

3. Liu M, Wilairat $\mathrm{P}$ and Go M L, J Med Chem., 2001, 44(25), 4443-4452; DOI:10.1021/jm0101747

4. Solankee A, Kapadia K, Solankee S and Patel G, J Ind Chem Soc., 2009, 86, 837.

5. Aponte J C, Verastegui M, Malaga E, Zimic M, Quiliano M, Vaisberg A J, Gilman R H and Hammond G B, J Med Chem., 2008, 51(19), 6230-6234; DOI:10.1021/jm800812k

6. Sabzevari O, Mahmoudian S, Minaei B and Paydar H, Toxicol Lett., 2010, 196, S213; DOI:10.1016/j.toxlet.2010.03.717

7. Powers D G, Casebier D S, Fokas D, Ryan W J, Troth J R and Coffen D L, Tetrahedron, 1998, 54(16), 4085-4096; DOI:10.1016/S0040-4020(98)00137-9

8. Amir M, Kumar H and Khan S A, Bioorg Med Chem Lett., 2008, 18(3), 918-922; DOI:10.1016/j.bmcl.2007.12.043

9. Yar M S, Siddiqui A A and Ali M A, J Serb Chem Soc., 2007, 72, 5-11.

10. Kelekci N G, Yabanoglu S, Kupeli E, Salgin U, Ozgen O, Ucar G, Yesilada E, Kendi E, Yesilada A and Bilgin A A, Bioorg Med Chem., 2007, 15(17), 5775-5786; DOI:10.1016/j.bmc.2007.06.004

11. Ozdemir Z, Kandilci H B, Gumusel B, Calis U and Bilgin A A, Eur J Med Chem., 2007, 42(3), 373-379; DOI:10.1016/j.ejmech.2006.09.006 
12. Thirunarayanan $\mathrm{G}, I L C P A, 2014, \mathbf{1 8}, 47$.

13. Solankee A., Kapadia K, Ciric A, Sokovic M, Doytchinova I and Geronikaki A, Eur J Med Chem., 2010, 45(2), 510-518; DOI:10.1016/j.ejmech.2009.10.037

14. Showalter H D H, Johnson J L, Werbel L M, Leopold W R, Jackson R C and Elslager E F, J Med Chem., 1984, 27(3), 253-255; DOI:10.1021/jm00369a002

15. Cho S, Kim S, Jin Z, Yang H, Han D, Baek N I, Jo J, Cho C W, Park J H, Shimizu M and Jin Y H, Biochem Biophys Res Commun., 2011, 413(4), 637-642; DOI:10.1016/j.bbrc.2011.09.026

16. Maya S C, Ortega S H, Apan T R and Lijanova I V, Bioorg Med Chem., 2012, 20(1), 415-421; DOI:10.1016/j.bmc.2011.10.070

17. Grossi G, Braccio M D, Roma G, Ballabeni V, Tognolini M, Calcina F and Barocelli E, Euro J Med Chem., 2002, 37(12), 933-944; DOI:10.1016/S0223-5234(02)01400-9

18. El-Subbagh H I, Hassan G S, El-Azab A S, Aziz A M A, Kadi A A, Al-Obaid A M, Al-Shabanah O A and Ahmed M M S, Eur J Med Chem., 2011, 46(11), 5567-5572; DOI:10.1016/j.ejmech.2011.09.021

19. Kumar R and Joshi Y C, J Serb Chem Soc., 2008, 73, 937-943.

20. Solankee A, Patel K and Patel R, J Chem., 2012, 9(4), 1897-1905; DOI:10.1155/2012/638452

21. Rattan A, In: Antimicrobials in Laboratory Medicine. $5^{\text {th }}$ Ed., B.Y. Churchill Livingstone, New Delhi, 2005, 85-90. 